\title{
A Novel Integrated High Precision Vacuum Microelectronic Accelerometer
}

\author{
Haitao Liu ${ }^{1, *}$, Zhiyu Wen ${ }^{1}$, Wengang Huang ${ }^{2}$, Dongling Li ${ }^{1}$, Li Chen ${ }^{1}$ and XiaoHan Yuan ${ }^{1}$ \\ 1 National Key Discipline Laboratory of Novel Micro/Nano Devices and Systems Technologies, Chongqing \\ University, Chongqing 400044, China; wzy@cqu.edu.cn (Z.W.); lidongling@cqu.edu.cn (D.L.); \\ cl2009@cqu.edu.cn (L.C.); yuanxiaohan2008@126.com (X.Y.) \\ 2 NO. 24 Research Institute of CETC, Chongqing 400060, China; hwg_cq@163.com \\ * Correspondence: htliu@cqu.edu.cn; Tel.: +86-23-65104131
}

\begin{abstract}
In this paper, a novel integrated high precision vacuum microelectronic accelerometer is put forward based on the theory of field emission, the accelerometer consists of sensitive structure and interface ASIC. The sensitive structure has a mass of a cathode cone tips array, a folded beam, an emitter electrode and a feedback electrode. The sensor is fabricated on a double side polished (1 00 ) N-type silicon wafer, the tips array of cathode are shaped by wet etching with HNA (HNO3, $\mathrm{HF}$ and $\mathrm{CH} 3 \mathrm{COOH}$ ) and metalized by TiW/Au thin film. The structure of sensor is released by ICP process finally. The interface ASIC was designed and fabricated based on the P-JFET high voltage bipolar process. The accelerometer is tested through static field rollover test, and the test results show the integrated vacuum microelectronic accelerometer has good performances, which sensitivity is $3.081 \mathrm{~V} / \mathrm{g}$ and non-linearity is $0.84 \%$ in the measuring range of $-1 \mathrm{~g} \sim \mathrm{g}$.
\end{abstract}

Keywords: field emission; integrated; vacuum microelectronic; cathode tips array; interface ASIC

\section{Introduction}

There is increasing demand toward small size, lightweight and low power sensing systems in micro accelerometers. More especially, MEMS (Micro-Electro Mechanical System)-based accelerometers find great applications in navigation systems [1-4], inertial sensors [5-7], seismometer [8], space microgravity [9], military affairs [10], and optical devices [11]. Since the world's first fieldbased sensor has been launched, field emission devices have been widely used due to their high accuracy, high sensitivity and anti-radiation advantages.

The vacuum microelectronics accelerometer is based on the field emission principle[12], while the field emission has two distinct advantages over other accelerometers due to the feature of the cold cathode emission[13-15], the output current signal of sensor changes exponentially with the acceleration, so the sensitivity is very high; and the current output of the sensor makes its interface current relatively simple. In this paper, a novel integrated high precision vacuum microelectronic accelerometer is proposed. This paper presents the principle and structure; and the design and fabrication of sensor and interface circuits; then demonstrates the experimental results of the circuits and accelerometer; and the conclusion is get finally.

\section{Principle and Structure}

The vacuum microelectronics accelerometer is based on the metal field emission principle that Metal field emission is a kind of electron emission phenomenon that relies on a strong external electric field to suppress the potential barrier of the metal surface, reduce the barrier and narrow the barrier width. The stronger the applied electric field is, the height of surface barrier is not only reduced, but also the width is narrowed. When the barrier width is narrow enough to be comparable to the electron wavelength, the electrons can escape through the potential barrier, thereby forming a field electron emission in vacuum. 
The metal field emission current can be calculated by the Fowler and Nordheim Formula (F-N) $[16,17]$. The simplify Fowler- Nordheim Formula is shown as equation (1).

$$
J=1.5 \times 10^{-6} \frac{E^{2}}{\phi} \exp \left(\frac{10.4}{\phi^{1 / 2}}\right) \exp \left(-16.44 \times 10^{7} \frac{\phi^{3 / 2}}{E}\right)
$$

Where, $\mathrm{J}$ is field emission current density $(\mathrm{A} / \mathrm{cm} 2), \mathrm{E}$ is surface electric field strength $(\mathrm{V} / \mathrm{cm})$, and $\ddot{o}$ is work function(eV). The surface electric field strength of a tip is shown as equation(2).

$$
E=\frac{2}{r \ln \left(\frac{d}{r}\right)} V
$$

Where $\mathrm{r}$ is the curvature radius of cone tip, $\mathrm{d}$ is the distance between the cathode cone and the anode plate, and $\mathrm{V}$ is the voltage applied on the anode.

The distance between the emission current density and the cone tip radius of curvature and the distance between the cathode cone and the anode plate can be obtained by calculation, as shown in Figure 1. and Figure 2. It can be seen from the figure that the emission current density increases exponentially with the radius of curvature of the cone tip, and the decrease in the distance from the tip to the anode plate at a certain emission voltage. In order for the field emission device to obtain a larger output current at a smaller operating voltage, the radius of curvature of the tip and the distance from the tip to the anode plate should be minimized.

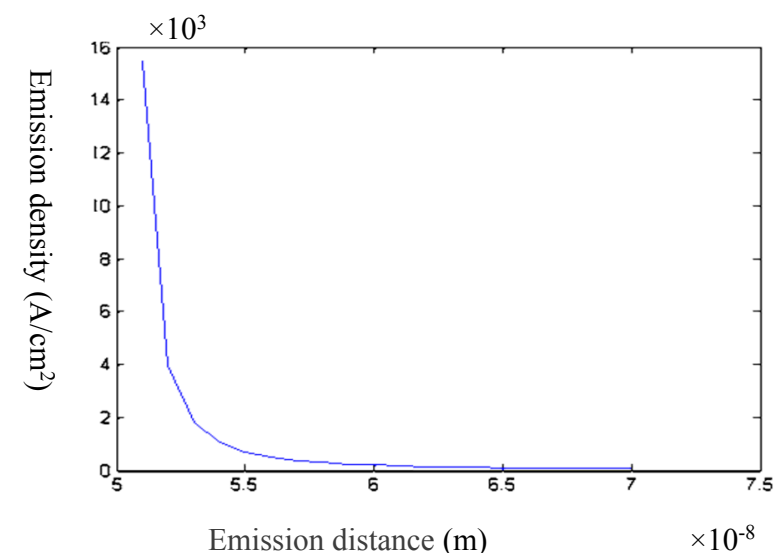

Figure 1. Emission current density vs. distance between cathode tips and anode.

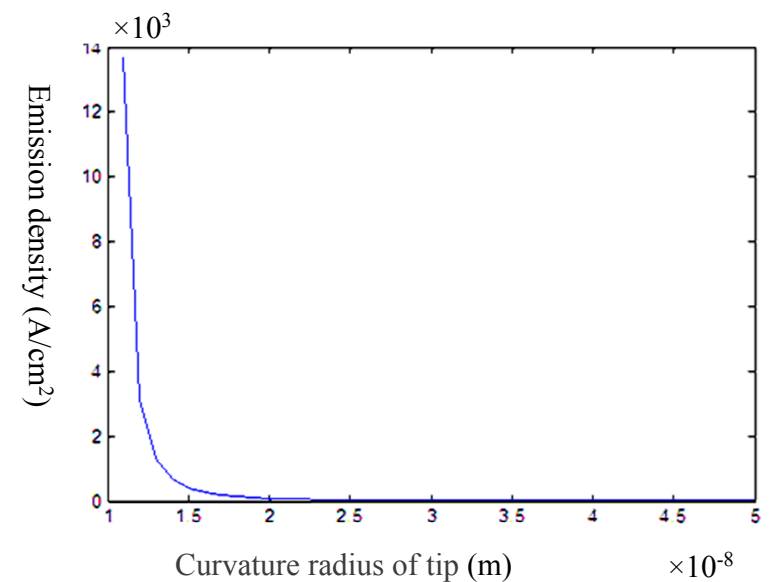

Figure 2. Emission current density vs. curvature radius of tip.

The structure of sensor consists of a proof mass with a field emission cathode tips array, cantilever beam, anode and feedback electrodes shown in Figure 3. And there are about 10000 tips array on the cathode, which make the emission current increased greatly. The external acceleration will lead to the proof mass moving and cause the distance between the cathode and anode tips array change, cause the cathode field emission current dramatic vary with exponential relationship, so the acceleration can be obtained by detecting the cathode emission current. 




Figure 3. Structure of accelerometer.

\section{Design and Simulations}

\subsection{Sensor sensitive unit}

The sensitive element designed by a cantilever-mass, the cantilever is a novel folded beam structure, shown in Figure 4. Based on the deformation energy analysis method, and mechanical and electric analysis, the rectangular folding beam elastic stiffness of vacuum microelectronic accelerometer is calculated [18]. The design parameters of structure are shown in Table 1.



(a)

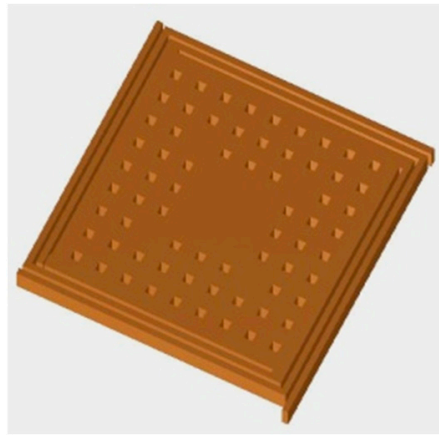

(b)

Figure 4. (a) Plan view of sensitive structure; (b) 3-D map view of sensitive structure.

Table1. Key structure parameters of sensor

\begin{tabular}{ll}
\hline \multicolumn{1}{c}{ Parameters } & value \\
\hline Beam Length & $3200 \mathrm{um}$ \\
Beam Width & 200um \\
Beam Thickness & $20 \mathrm{um}$ \\
Mass Side Length & $2600 \mathrm{um}$ \\
Mass Thickness & 80um \\
\hline
\end{tabular}

The resonant frequency and modal response of the sensor are analyzed by finite element simulation(FEM), the analysis results by ANSYS are shown in Figure 5 and Table 2, the resonant frequency is $979.08 \mathrm{~Hz}$, and the 2nd mode is $1774.5 \mathrm{~Hz}$ and the $3 \mathrm{rd}$ mode is $1774.7 \mathrm{~Hz}$, and the 4 th mode is $3069.8 \mathrm{~Hz}$. 

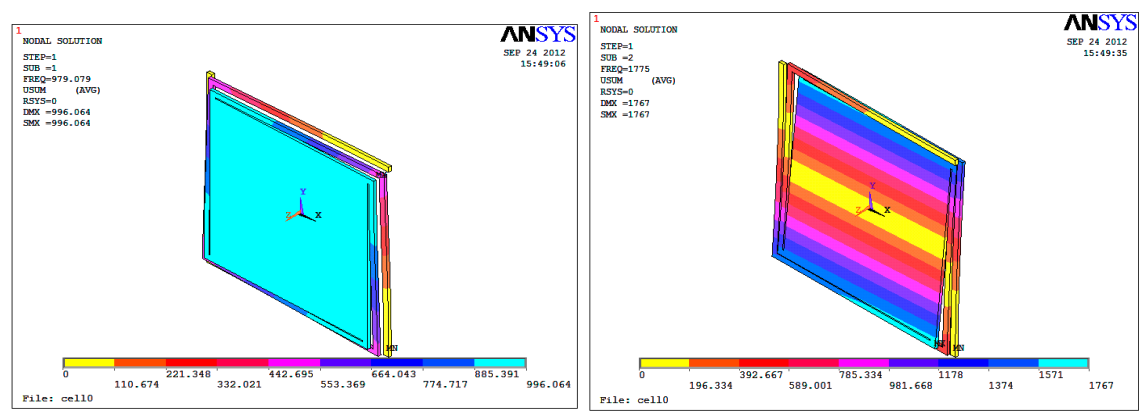

(a)

(b)



(c)



(d)

Figure 5. (a) 1st mode analysis results; (b) 2nd mode analysis results; (c) 3rd mode analysis results; (d) 4th mode analysis results.

Table 2. Results of mode analysis of accelerometer

\begin{tabular}{ccccc}
\hline SET & FREQ(Hz) & LOADSTEP & SUBSTEP & CUMULATIVE \\
\hline 1 & 979.08 & 1 & 1 & 1 \\
2 & 1774.5 & 1 & 2 & 2 \\
3 & 1774.7 & 1 & 3 & 3 \\
4 & 3069.8 & 1 & 4 & 4 \\
\hline
\end{tabular}

The elastic stiffness of the sensor is calculated by static simulation, the analysis result is shown in Figure 6. When $\mathrm{a}+1 \mathrm{~g}$ acceleration is applied along the $\mathrm{z}$-axis direction, the mass will move by 1.3 $\mu \mathrm{m}$, the effect force applied on the proof mass is $4.72 \times 10-5 \mathrm{~N}$, so the approximate elastic stiffness is $36 \mathrm{~N} / \mathrm{m}$ 。



Figure 6. Displacement contour under $+1 \mathrm{~g}$ acceleration. 


\subsection{Process and Fabrication}

The vacuum microelectronic accelerometer is possessed by bulk silicon MEMS technology based on the double side polished $\mathrm{N}$-type $\left(\begin{array}{lll}1 & 0 & 0\end{array}\right)$ silicon wafer, which has high tensile strength and low mechanical losses. The process of fabrication of vacuum microelectronic accelerometer includes oxidation, magnetron sputtering, ion implantation, anodic bonding, ICP and other key processing technology as shown in Figure 7. The silicon tips array are form by wet etching with HNA (HNO3, $\mathrm{HF}$ and $\mathrm{CH} 3 \mathrm{COOH}$ ) and metalized by TiW/Au thin film, the morphology of the tip is an ideal pyramid as shown in Figure 8.

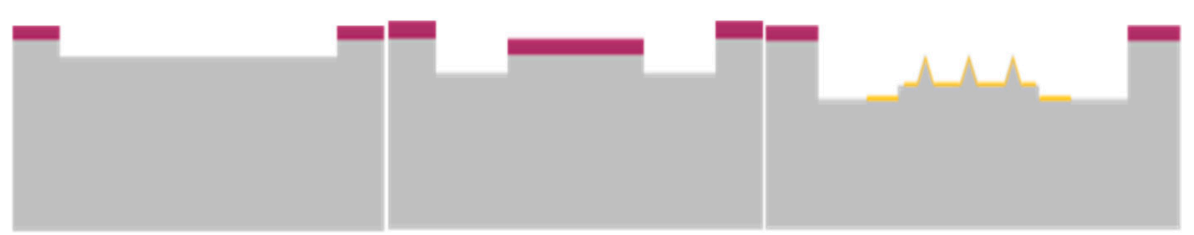

(a)

(b)

(c)



(d)

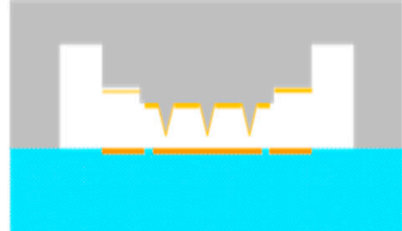

(g) (e)

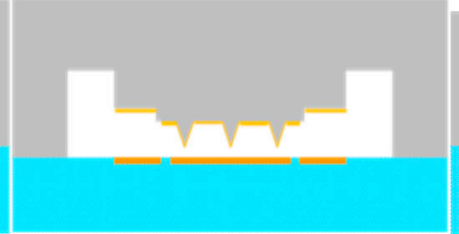

(h) (f)

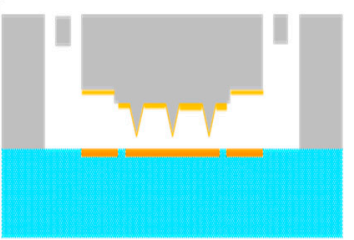

(i)

Figure 7. Process of vacuum microelectronic accelerometer sensor. (a) Etching grooves window to form a bonded anchor; (b) Etching grooves window to form cone station; (c) Corrosion cone, and sharpening; (d) After ion implantation, cone metallization; (e) ICP etching front to form beam area; (f) Growth electrodes on glass; ( $g$ ) Bonding the silicon and glass; ( $h$ ) The silicon is thinned by $\mathrm{KOH}$ etching; (i) ICP structure release to form beam.

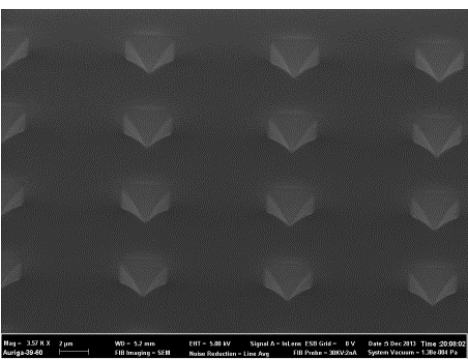

(a)

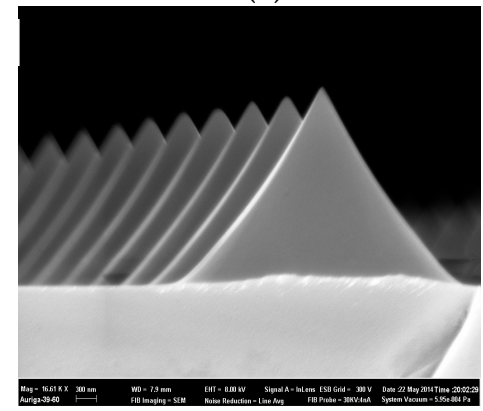

(c)

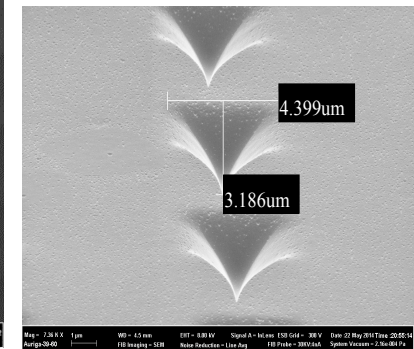

(b)

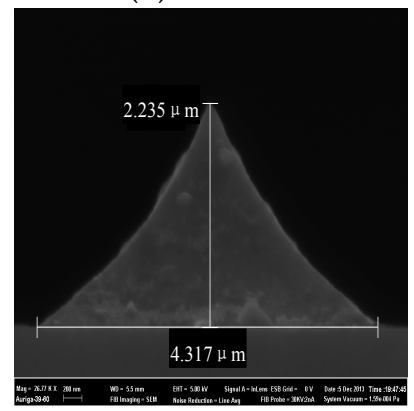

(d)

Figure 8. SEM of cathode tips array. (a)array front view; (b) partial front view; (c) Array Side view; (d) Partial Side view. 
The cathode cone tip surface of vacuum microelectronic accelerometer is easily oxidized under low vacuum, which led to the emission current drop and instability, in order to improve the emission current stability of vacuum microelectronic acceleration sensor, the surface was coated a layer composite metal film to protect the cone tip which can effectively improve the stability of the emission current.

\subsection{Interface Circuit}

The control and detecting interface ASIC of vacuum microelectronic accelerometer was designed based the PJFET high voltage bipolar process of CETC (China Electronic Technology Group) No. 24 institute, the circuits mainly include two cascade operational amplifier shown in Figure 9.



Figure 9. Schematic of ASIC circuit.

The sensor cathode plate is connecting to the grounded, and the discharge current is controlled by excitation voltage VR1, Rs is used to adjust the sensor resistance. Amplifier U1A is work as a transimpedance amplifier, the cathode discharge current is converted to output voltage signal through the trans-impedance amplifier, and the emission current is calculated as shown in equation (3):

$$
I=\frac{V R 2-\operatorname{VOS} 2-(V R 1-\operatorname{VOS} 1)}{R F 1}
$$

Where, VOS1 and VOS2 are input offset voltage of the two amplifiers respectively. The value of current I can be changed by adjusting RF1 or VR2.

The amplifier U2B realizes PI control link to provide a high DC gain of system to ensure the high accuracy, but not produces low frequency pole affect to ensure the system stability. Bias voltage is provided by VR2, resistors R1 R3 adjust the output voltage range of the amplifier U2B to prevent the feedback voltage VF from being too large to cause the sensor to saturate. In order to keep the loop stable, the main pole frequency must be low enough to keep away from the main pole. The main pole is set at the trans-impedance amplifier (A1) by increasing the CF1.

The basic structure of the operational amplifier U1 is shown in Figure 10, Figure 11 is schematic Circuit of the interface ASIC.

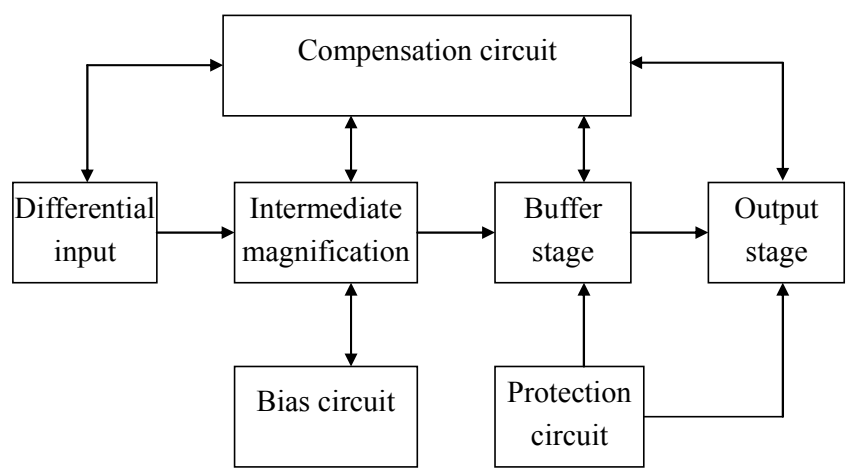

Figure 10. Basic structure of the ASIC. 


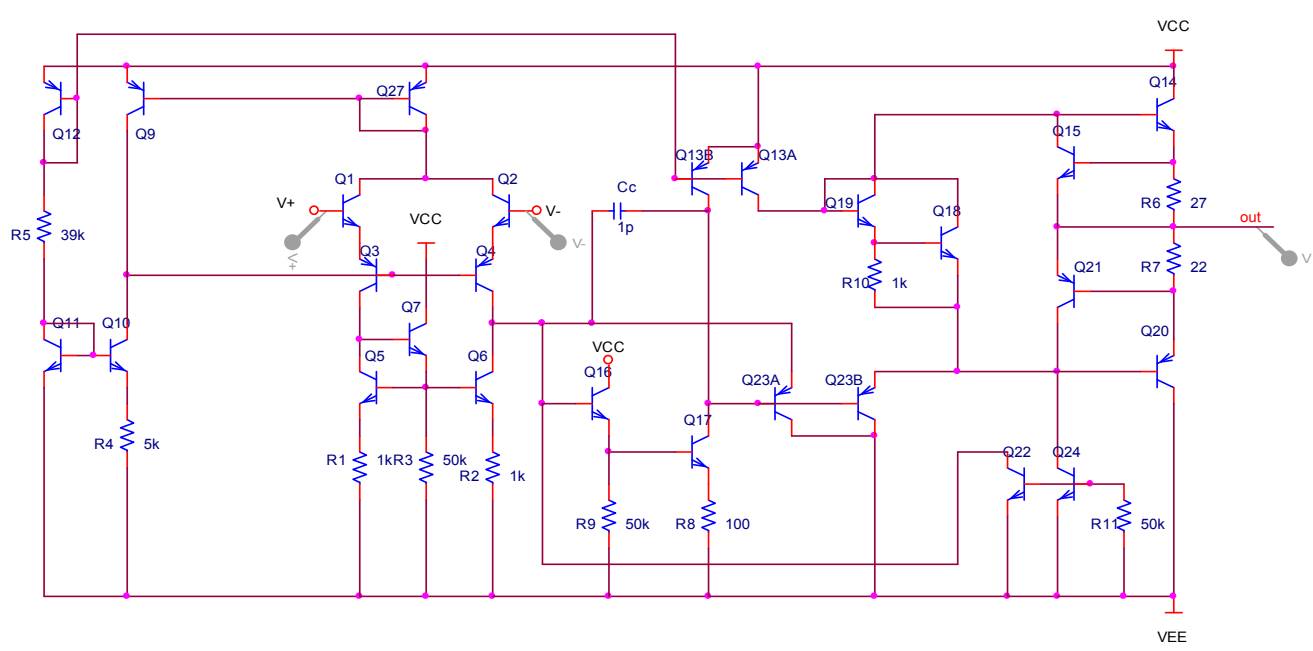

Figure 11. Schematic Circuit of the ASIC.

Finally, the ASIC was fabricated based the P-JFET high voltage bipolar process in the china CETC 24 research institute. Figure 12-15 are the layout of the basic component unit of interface ASIC.



(a)

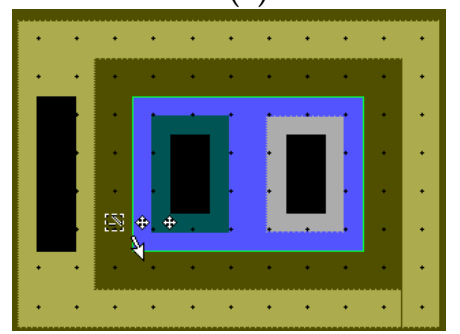

(c)

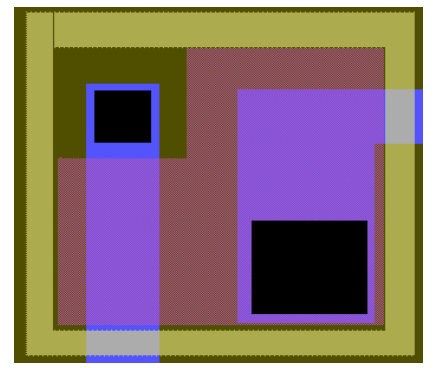

(b)



(d)

Figure 12. (a) Resistor unit layout; (b)MOS capacitor layout; (c) NPN transistor layout; (d)Substrate lateral PNP transistor layout.

\section{Tests and Results}

Finally, the sensitive structure unit and interface ASIC chip was integrated on a PCB board, the photo of integrated vacuum microelectronic accelerometer is shown in Figure 13, and the working voltage of accelerometer is $\pm 15 \mathrm{~V}$ power supply. Table 3 shows the test results of main parameter of the interface ASIC. 


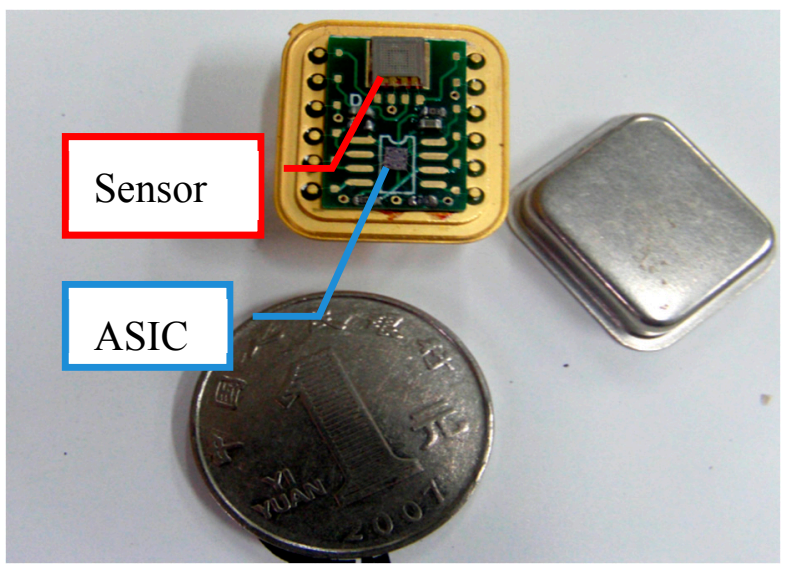

Figure 13. Photo of integrated vacuum microelectronic accelerometer.

Table 3. Main parameter of ASIC

\begin{tabular}{llllllll}
\hline Parameters & Vos $(\mathrm{mV})$ & $\mathrm{Ib}+(\mathrm{nA})$ & $\mathrm{Ib}-(\mathrm{nA})$ & $\operatorname{Ios}(\mathrm{nA})$ & Avo(db) & CMRR(db) & PSRR(db) \\
\hline $\begin{array}{l}\text { Design } \\
\text { specifications }\end{array}$ & $<5$ & $<150$ & $<150$ & $<30$ & $>96$ & $>70$ & $>65$ \\
Test Results & -1.29 & -16.3 & -15.8 & -0.5 & 103 & 81 & 106 \\
\hline
\end{tabular}

Gravitational field static rollover test was carried to test the sensitivity, linearity, zero stability use the mirroring precision rotary indexing head. Testing data are shown in Figure 14 and Table3. Test results show that the measuring range $-1 \mathrm{~g} 1 \mathrm{~g}$, and sensitivity of accelerometer is $3.081 \mathrm{~V} / \mathrm{g}$, the least squares fitting correlation coefficient reaches 0.99998 , non-linearity is $0.84 \%$, and zero stability is $0.4 \mathrm{mg}$.

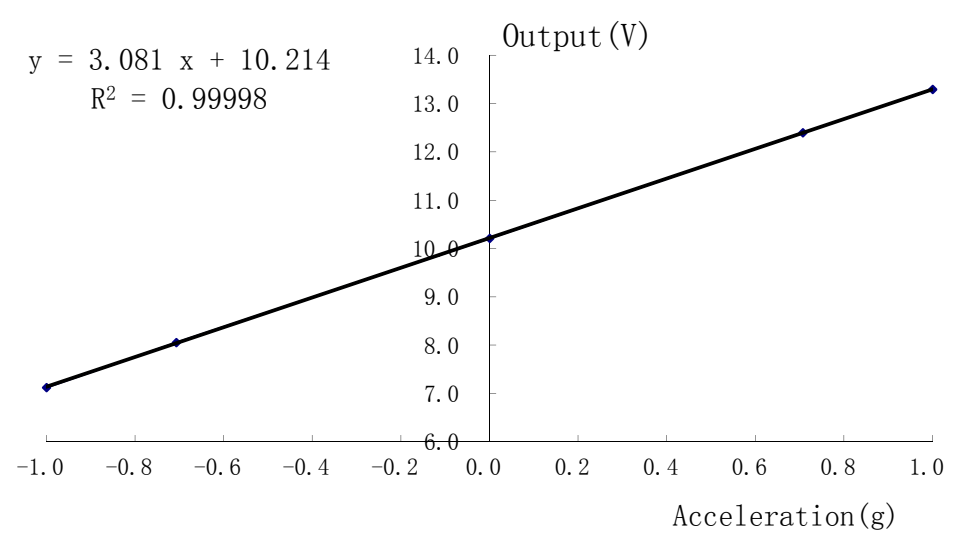

Figure 14. Curve of output data vs. input.

Table 3. Datum of static gravitational field roll test

\begin{tabular}{|c|c|c|c|c|}
\hline $\begin{array}{l}\text { Acceleration. (g } \\
\text { ) }\end{array}$ & $\begin{array}{l}\text { Measure } \\
\text { value (V } \\
)\end{array}$ & $\begin{array}{l}\text { Fitting } \\
\text { value (V } \\
\text { ) }\end{array}$ & $\begin{array}{l}\text { Deviation（V } \\
\text { ) }\end{array}$ & $\begin{array}{l}\text { Non- } \\
\text { linearity. }\end{array}$ \\
\hline+1.0 & 13.292 & 13.314 & -0.022 & $-0.71 \%$ \\
\hline+0.7 & 12.397 & 12.411 & -0.014 & $-0.46 \%$ \\
\hline 0 & 10.206 & 10.230 & -0.024 & $-0.76 \%$ \\
\hline-0.7 & 8.053 & 8.049 & 0.004 & $0.12 \%$ \\
\hline-1.0 & 7.120 & 7.146 & -0.026 & $-0.84 \%$ \\
\hline
\end{tabular}




\section{Conclusions}

Vacuum microelectronic accelerometer is based on field emission and has many advantages of anti-radiation, high sensitivity and high accuracy. This paper presents the principle, design, fabrication, interface ASIC and tests of a novel integrated high precision vacuum microelectronic accelerometer. Test results of accelerometer show that the sensitivity is about $3.081 \mathrm{~V} / \mathrm{g}$ and nonlinearity is about $0.84 \%$ over a range of $-1 \mathrm{~g} \sim \mathrm{g}$. It can be widely used in acoustic measurement, navigation, earthquake monitoring and aerospace and so on.

Acknowledgments: The work was supported by the Fundamental Research Funds for the Central Universities of China under Grant 106112014CDJZR160001, the Common Key technology innovation projects in key industries of Chongqing under Grant cstc2016zdcy-ztzx0034 .

Author Contributions: Liu H.T. and Wen Z.Y. conceived and designed the sensor; Li D.L. performed the process; Chen L. performed the experiments; Huang W.G. designed the circuit; Liu H.T. wrote the paper.

Conflicts of Interest: The authors declare no conflict of interest.

\section{References}

1. Syed, Z.; Aggarwal, P.; Niu, X.; Sheimy,N. Economical and robust inertial sensor configuration for a portable navigation system. Physics Letters A 2007, 365(4), 263-267.

2. Mougenot, D.; Thorburn, N. MEMS-based 3D accelerometers for land seismic acquisition: is it time? Leading Edge 2004, 23, 246-250.

3. Monajemi, P.; Ayazi, F. Design optimization and implementation of a micro-gravity capacitive HARPSS accelerometer, IEEE Sens 2006, 6 , 39-46.

4. Dong Y.G.; 2007. MICROSENSOR; Tsinghua University Press: Beijing, China, 2007.

5. King, K.; Yoon, S.W.; Perkins N.C.; Najafi K. Wireless MEMS inertial sensor system for golf swing dynamic. Sensors \& Actuators A Physical 2008, 141(2), 619-630.

6. S. Nakamura. MEMS inertial sensor toward higher accuracy \& multi-axis sensing. Sensors 2005, 4, 939-942

7. Sun,C.M.; Tsai,M.H.; Liu,Y.C.; Fang, W. Implementation of a monolithic single proof-mass tri-axis accelerometer using CMOS-MEMS technique. IEEE Trans. Electron Devices 2010, 57 ,1670-1679.

8. Ding H. G., Advances trends and recommendations in micro-nano technology. Nanotechnology and Precision Engineering 2006, 4 (4), 249-255.

9. Tan X. Y. Liu X. W.; Development of Small satellite and Micro-satellite Speed up By MEMS Technology. Chinese Journal of Scientific Instrument 2004, 25(4), 598-600.

10. Ruffin, P.B.; Burgetr, S. J. Recent progress in MEMS technology development for military application, Processing of SPIE 2001, 4334, 1-12.

11. Wu, M.C.; Solgaard, O.; Ford, J.E. Optical MEMS for lightwave communication, Journal of Lightwave Technology 2006, 24(12), 4433-4454.

12. Xia, S. H. Research and Development of Vacuum Microelectronic Sensors, Journal of Mechanical Strength 2001, 23, 535-538.

13. Peng, S. C., Wen, Z. Y.; Wen Z.Q.; Pan Y.S.; LI Xia. Finite Element Analysis on Vacuum Microelectronic Acceleration Sensor, Micronanoelectronic Technology, 2003, 7; 292-301.

14. Paul, J., Anthony, J. K., A Planar CMOS Field-Emission Vacuum Magnetic Sensor, IEEE Transactions on Electron Devices, 2009, 56(4), 692-695.

15. $\mathrm{Xu}, \mathrm{S}$. L. Master Degree, Study on vacuum microelectronic pressure sensor, Chongqing University, Chongqing, China, 2003.

16. Wang B. P. Vacuum microelectronics and its application. Southeast University Press: Nanjing, China, 2002.

17. Neamen, A.D. Semiconductor Physics and Devices, 3rd Ed.; Electronic Industry Press: Beijing, China, 2005.

18. Liu H.T. ; Wen Z.Y.; Shang Z.G. ; Chen L. A new method to analyze the stiffness of MEMS accelerometer. Key Engineering Material 2014, 609-610, 710-714. 